\title{
SÍFILIS CONGÉNITA
}

\author{
Carlos Velásquez Vásquez ${ }^{1}$
}

\begin{abstract}
RESUMEN
Con la importancia que adquirió la lucha mundial contra el virus de inmunodeficiencia humana, la sífilis congénita (SC) se ha mantenido como una epidemia silenciosa que ha estado incrementando los indicadores de mortalidad y morbilidad fetal/neonatal. Epidemiológicamente los mayores índices de SC se encuentran en países donde el sistema sanitario no es el más adecuado para la atención de la madre y el niño de allí que se hable que la tasa de SC es un indicador negativo de los sistemas de salud. Algunas condiciones, como la dificultad de su diagnóstico y los cambios de criterios para su determinación, no han permitido tener información confiable acerca de su impacto, aunque es evidente que países del tercer mundo sobre todo de África Subsahariana se ven más afectados por esta. La OMS estima que cada año es responsable de 460 mil abortos o natimuertos, 270 mil casos de sífilis congénita y el nacimiento de 270 mil nacimientos prematuros o de bajo peso. La SC puede manifestarse en muerte fetal con aborto espontáneo después del primer trimestre de gestación, o natimuertos en un 30 a $40 \%$; o puede culminar en el parto de un recién nacido prematuro con evidentes signos clínicos de infección; o nacer completamente asintomático. Su presentación clínica va a depender del grado de compromiso orgánico por la respuesta inmunológica al treponema desde la etapa fetal. La terapia de elección es la penicilina, pero la mejor forma de eliminarla es el diagnóstico y tratamiento de la sífilis materna durante el embarazo.
\end{abstract}

Palabras clave: Sifilis congénita; Sifilis Latente; Muerte fetal; Prematuro (fuente: DeCS BIREME).

\section{CONGENITAL SYPHILIS}

\begin{abstract}
With the large development of the global fight against human immunodeficiency virus, congenital syphilis (CS) has remained a silent epidemic that has seen increasing mortality and morbidity indicators fetal / neonatal. Epidemiologically the highest rates of CS are in countries where the health system is not the most suitable for the care of the mother and child from there to talk to the CS rate is a negative indicator of health systems. Some conditions, such as the difficulty of diagnosis and changes in criteria for determination, not allowed to have reliable information about its impact, although it is clear that third world countries especially sub-Saharan Africa are most affected by this. WHO estimates that each year is responsible for 460,000 abortions or stillbirths, 270000 cases of congenital syphilis and the birth of 270000 premature or low birth weight. The SC can manifest in fetal death and spontaneous abortion after the first trimester of pregnancy, or stillbirth by 30 to $40 \%$, or can culminate in the birth of a premature infant with obvious clinical signs of infection, or birth completely asymptomatic. Clinical presentation will depend on the degree of organ involvement by the immune response to Treponema from the fetal stage. The treatment of choice is penicillin, but the best way to eliminate the diagnosis and treatment of maternal syphilis during pregnancy
\end{abstract}

Key words: Congenital syphilis; Latent syphilis; Stillbirth; Premature (source: MeSH NLM).

\section{INTRODUCCIÓN}

La sífilis congénita, también llamada intrauterina o prenatal, es aquella que afecta al feto desde la vida intrauterina. Actualmente es considerada un problema de salud pública debido a los altos indicadores de mortalidad fetal y neonatal en los países del tercer mundo producto de la infección materna no diagnosticada o tratada oportunamente, lo que traduce un inadecuado sistema sanitario donde falla una de las principales actividades dirigidas a la protección de la salud materna y neonatal como es el adecuado control prenatal. Con la importancia que tomó la infección por VIH en las últimas décadas, el control de la sífilis y por ende de la sífilis congénita disminuyó ostensiblemente lo que ha permitido el resurgimiento de mayor cantidad de casos a pesar de la facilidad que existe en el diagnóstico y tratamiento. El artículo busca revisar algunos aspectos de la sífilis congénita que nos permita recordar su presencia y la necesidad de emprender todos los esfuerzos posibles para su eliminación en beneficio de nuestros niños.

\section{EPIDEMIOLOGÍA}

Las infecciones de transmisión sexual (ITS) han repercutido desde el inicio de la historia del hombre de manera significativa en la vida individual y colectiva de la sociedad. Tal es el caso de la sífilis que en el transcurrir de la historia ha sido causa de verdaderas epidemias que han afectado a grandes masas de la población europea, medio-oriental y americana, con consecuencias devastadoras. A pesar de las controversias del origen de la sífilis, de manera inobjetable se conoció por su contundencia en Europa a fines del siglo XV, convirtiéndose en una rápida epidemia multinacional donde

Médico Pediatra. Departamento de Neonatología. Instituto Nacional Materno Perinatal. Lima-Perú. 
el aspecto sexual era el predominante y con características clínicas que la hicieron rápidamente conocida y temida ${ }^{1}$

Aunque persiste la controversia en torno a si fueron realmente los españoles quienes llevaron la sífilis al viejo continente, hay indicios de que Cristóbal Colón padeció la enfermedad y murió de ella en 1506 en Valladolid (España). A lo largo de la historia, muchos personajes, como Beethoven, Donizetti, Schubert, Schumann, Van Gogh y Hitler, han padecido de sífilis. Las implicaciones sociales de la enfermedad han llevado a ocultar el diagnóstico y a achacar a otras causas los síntomas neurológicos propios de la afección en sus etapas avanzadas.

El mercurio y el arsénico fueron dos de los remedios más utilizados para el tratamiento de la sífilis, y también de los más temidos, debido a las complicaciones que producían. Aunque August von Wassermann inventó la primera prueba de sangre para detectar la sífilis, no se contó con un tratamiento eficaz hasta 1943, cuando la penicilina, descubierta en 1928 por el bacteriólogo británico Alexander Fleming, se utilizó por vez primera con buenos resultados para tratar la enfermedad ${ }^{2}$.

Los seres humanos son los únicos hospederos de la sífilis. La enfermedad es más común en África SubSahariana, en el Sur y Sur Este de Asia y en América del Sur, con más de 3 millones de adultos infectados anualmente en estas regiones, en comparación de los 100,000 casos diagnosticados en Estados Unidos ${ }^{3}$.

La sífilis tiende a acompañar a poblaciones que se encuentran bajo cambios estresantes; de allí que son amplios los datos de masivas infecciones con sífilis durante la segunda guerra mundial en los países involucrados y también durante la crisis económica mundial de $1929^{4}$. Los factores de riesgo asociados con la adquisición de sífilis incluyen a la pobreza, uso de drogas (crack, cocaína), comercio sexual por drogas y dinero y la infección por VIH.

La Organización Mundial de la Salud (OMS) estima que 3.3 millones de niños nacen muertos al año, y más de 4 millones fallecen antes de los primeros 28 días de vida ${ }^{5}$. La sífilis congénita representa una importante causa de mortalidad en este período, algunos reportes refieren que puede significar alrededor del $26 \%$ de los natimuertos ${ }^{6}$.

El rol que tienen las enfermedades específicas, como la sífilis, en la mortalidad de los recién nacidos es difícil de estimar. Schmidt y colaboradores, utilizaron datos publicados en la literatura respecto a la prevalencia de sífilis materna y de los reportes de sífilis congénita, para realizar las estimaciones acerca de la tasa mundial de estas enfermedades. Sus hallazgos sugirieron que existen aproximadamente 2 millones de sífilis materna cada año, y entre 728,547 y $1,527,560$ de sífilis congénita anualmente ${ }^{7}$.

El cambio de definición de caso de sífilis congénita varió de los criterios clásicos de Kauffman a los sugeridos por
Tabla 1. Criterios diagnóstico de sífilis congénita

\begin{tabular}{|c|c|}
\hline Criterios de Kauffman & Criterios de la CDC \\
\hline - Definitivo & - Confirmado \\
\hline $\begin{array}{l}\text { Treponema pálido por } \\
\text { campo oscuro o hallazgo } \\
\text { histológico }\end{array}$ & $\begin{array}{l}\text { Demostración por laboratorio } \\
\text { del Treponema }\end{array}$ \\
\hline \multirow{7}{*}{$\begin{array}{l}\text { - Probable } \\
\text { Incremento del título de } \\
\text { VDRL en } 3 \text { meses o test } \\
\text { serológico que no revierte } \\
\text { después de } 4 \text { meses } \\
\text { Un criterio mayor o dos } \\
\text { criterios menores y un test } \\
\text { serológico para sífilis posi- }\end{array}$} & - Presuntivo \\
\hline & $\begin{array}{l}\text { Todo niño con sífilis no tratada } \\
\text { o inadecuadamente tratada }\end{array}$ \\
\hline & $\begin{array}{l}\text { Todo niño con prueba serológi- } \\
\text { ca positiva y hallazgos compa- }\end{array}$ \\
\hline & $\begin{array}{l}\text { tibles con sífilis; VDRL en LCR } \\
\text { o LCR con células }>5 \text { o proteí- } \\
\text { nas }>50 \text { sin otra causa }\end{array}$ \\
\hline & Test de Ig para sífilis reactivo \\
\hline & NATIMUERTO SIFILITICO \\
\hline & $\begin{array}{l}\text { Muerte fetal mayor de } 6 \text { sema- } \\
\text { nas o peso mayor de } 500 \mathrm{gr} \text { de } \\
\text { madre con sífilis no tratada o } \\
\text { inadecuadamente tratada }\end{array}$ \\
\hline \multicolumn{2}{|l|}{ - Posible } \\
\hline \multicolumn{2}{|l|}{$\begin{array}{l}\text { Un test de FTA reactivo sin } \\
\text { criterios clínicos }\end{array}$} \\
\hline
\end{tabular}

Criterios mayores: condiloma lata, osteocondritis, periostitis, rinitis hemorrágica. Criterios menores: cicatrices en los labios, lesiones mucocutaneas, hepatomegalia, espleomegalia, linfoadenopatía, anemia hemolítica, signos neurológicos.

el centro de Control de Infecciones (CDC), en 1988, este cambio de criterios diagnósticos originó variaciones en las tasas de la enfermedad entre los países que actualmente han sido resueltas. Los criterios de la CDC incluyen a todos los recién nacidos con evidencia clínica de sífilis activa como aquellos que no tienen signología pero que son hijos de madre con sífilis no tratada o inadecuadamente tratada ${ }^{8}$ En la tabla 1 se pueden observar algunas de las diferencias entre los criterios de Kauffman y de la CDC para la definición de caso de sífilis congénita.

Al utilizar los criterios de la CDC se incrementa la sensibilidad de los reportes de casos de sífilis congénita, aunque se incluyen casos de alto riesgo pero que no necesariamente se encuentran infectados; sus efectos en la vigilancia de la incidencia de sífilis congénita, pueden ser apreciados en las estadísticas reportadas en EE.UU. donde se observa un incremento importante del número de casos después del cambio de criterios para definir al caso de sífilis congénita, pero que después, gracias a sus intervenciones de salud pública, se puede apreciar una pendiente que permite valorar la eficiencia de estas intervenciones ${ }^{9}$ (figura 1).

\section{La definición de caso varió en EEUU en el año 1998.}

La tasa de sífilis congénita está relacionada directamente a la tasa de sífilis en mujeres en edad fértil. La seroprevalencia en el embarazo es bastante baja en países desarrollados; su rango varía de 0.002 en Europa a 4,3 en algunas regiones de EEUU ${ }^{10}$. Sin embargo pocas de estas infecciones culminan en sífilis congénita debido a que cuentan con una atención 


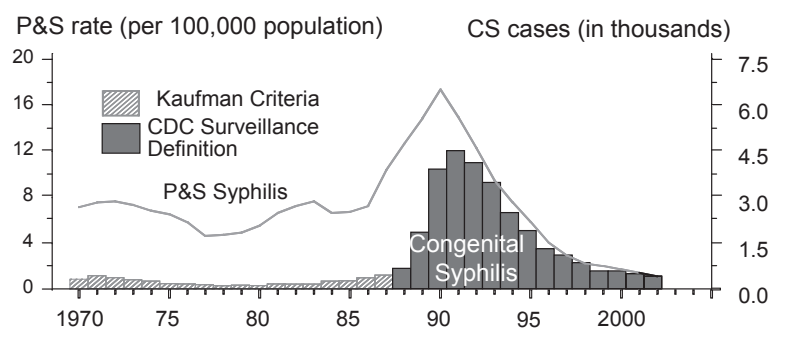

Figura 1. Casos de sífilis congénita según criterios de diagnóstico. EEUU 1970-2002

prenatal muy eficiente. Por ejemplo en EEUU se reportó 13.4 casos de sífilis por 100,000 recién nacidos vivos, en el año $2000{ }^{11}$, mientras que en el Reino Unido se reportaron 9 casos de sífilis congénita presuntiva entre 1994 y $1997^{12}$. En contraste existe un dramático incremento de las tasas de sífilis congénita en zonas rurales de Europa del Este y Asia ${ }^{13}$. En África se reporta niveles altos de tasas de prevalencia de sífilis materna en forma consistente $((3-18 \%)$ en las clínicas prenatales, por lo que las tasas de sífilis congénita son mayores del 1 por ciento en las guarderías infantiles ${ }^{14}$.

En la región de América Latina y el Caribe, la sífilis es la ITS de menor prevalencia entre las ITS clásicas. Es decir, la prevalencia de sífilis marca el límite inferior del espectro de prevalencia de todas las ITS. Según los datos suministrados a la Organización Panamericana de la salud (OPS) por los programas nacionales de ITS/VIH/SIDA durante el año 2004, la prevalencia estimada de sífilis en embarazadas es de $3,1 \%$ en la Región y oscila entre $1,00 \%$ en Perú y $6,21 \%$ en Paraguay. Según esos datos, la incidencia de sífilis congénita varía desde 1,40 por 1000 nacidos vivos en El Salvador, hasta 12 por 1000 nacidos vivos en Honduras, mientras que en Estados Unidos es de 0,10 casos por 1000 nacidos vivos ${ }^{15}$.

La OMS estima que cada año la sífilis materna es responsable de 460,000 abortos o natimuertos, 270,000 casos de sífilis congénita y el nacimiento de 270,000 nacimientos prematuros o de bajo peso. Hay datos limitados de las consecuencias de la sífilis materna no tratada en el feto y el neonato. Los resultados adversos del embarazo son 12 veces más en mujeres con sífilis que en la mujer seronegativa ${ }^{16}$.
Varios estudios hacen mención acerca de la frecuencia de efectos adversos de la sífilis materna no tratada, lo cierto es que estos efectos adversos promedian un $65 \%$ de los productos de madre con sífilis. Con estas estimaciones se puede realizar una aproximación del impacto de la sífilis materna sobre la morbimortalidad perinatal (Tabla 2).

La prevención de la sífilis congénita está basada en el tamizaje de sífilis en la gestante y la provisión oportuna del tratamiento con penicilina a la mujer infectada, la cual es altamente efectiva, segura y la única recomendación para toda gestante con sífilis ${ }^{17}$.

\section{LA PRESENTACIÓN CLÍNICA DE LA SÍFILIS CONGÉNITA}

Para poder comprender las diferentes formas clínicas en que se presenta la sífilis congénita, que varía desde la muerte fetal hasta la ausencia de sintomatología en el neonato, es necesario comprender algunos factores que influyen en la presencia o ausencia de ellos. La infección fetal es el resultado de la propagación hematógena del treponema desde una madre infectada; esta transmisión puede darse también en el momento del parto como resultado de contacto directo del recién nacido con lesiones genitales infecciosas de la madre. La propagación hematógena depende de la aparición de espiroquetas circulantes en sangre materna (espiroquetemia). Los estadíos tempranos de la sífilis se caracterizan por alta concentración de espiroquetemia, por lo que la probabilidad de infección al feto si la madre tiene sífilis temprana, es cercana al $100 \%$.

En el pasado se suponía que los treponemas no cruzaban la placenta hasta después de las 20 semanas de gestación. Los investigadores consideraban que la capa de células de Langhans del citotrofoblasto constituía una barrera placentaria eficaz contra la infección durante las primeras semanas. Sin embargo, esta teoría tuvo que ser descontinuada una vez que se descubrió que la capa de células Langhans persistía durante todo el embarazo ${ }^{19}$ y se encontraba en mujeres que finalmente transmitieron el treponema al feto. Además, en 1974, Harter Benirscheke examinó tejido fetal de abortos espontáneos de mujeres con sífilis, identificando espiroquetas en abortos de 9 y 10 semanas de gestación ${ }^{20}$.

Tabla 2. Eventos adversos en la culminación del embarazo de gestantes con sífilis. Diferentes autores.

\begin{tabular}{|c|c|c|c|c|c|c|}
\hline & $\begin{array}{c}1917 \\
\text { Harnman }\end{array}$ & $\begin{array}{c}1951 \\
\text { Ingraham }\end{array}$ & $\begin{array}{c}1987 \\
\text { Schulz }\end{array}$ & $\begin{array}{l}1990 \\
\text { Hira }\end{array}$ & $\begin{array}{l}2000 \\
\text { Global Burden of STI }\end{array}$ & $\begin{array}{c}2000 \\
\text { Watson-Jones }\end{array}$ \\
\hline Aborto & $17 \%$ & $22 \%$ & $30-40 \%$ & $22 \%$ & $20 \%$ & $25 \%$ \\
\hline Natimuertos & $23 \%$ & $12 \%$ & $10-20 \%$ & No datos & $15 \%$ & No datos \\
\hline Sífilis congénita & $21 \%$ & $33 \%$ & $10-20 \%$ & $2 \%$ & $20 \%$ & No datos \\
\hline Prematuridad & No datos & No datos & No datos & $33 \%$ & $20 \%$ & $25 \%$ \\
\hline Algún efecto adverso & $61 \%$ & $67 \%$ & $50-80 \%$ & $57 \%$ & $75 \%$ & $49 \%$ \\
\hline
\end{tabular}

Tomado de The Global elimination of congenital syphilis : rationale and strategy for action. World Health Organization 2007 


\section{Daño fetal}

Aunque en la actualidad es evidente que los treponemas atraviesan la placenta en la gestación temprana, hay pocas pruebas de los efectos adversos que pueda producir este hecho en el feto. Las lesiones encontradas en los fetos de madre sifilítica se caracterizan por infiltración perivascular por linfocitos, células plasmáticas e histiocitos, con endarteritis y extensa de fibrosis. Estas lesiones típicas reflejan una respuesta inflamatoria, y algunos investigadores han sugerido un importante papel de las citoquinas en la fisiopatología ${ }^{21}$. Trabajos adicionales sugieren que la interacción del T. pallidum con el endotelio vascular podría ser un importante evento temprano en la respuesta inmune cuyo resultado es el inicio de la cascada inflamatoria ${ }^{22} 23$.

La patología sugiere la participación de varios órganos, con una extensa respuesta inflamatoria. Las manifestaciones reflejan, en parte, la madurez inmunológica del feto una observación que fue formulada por Silverstein hace más de 40 años $^{24}$. En general, no es hasta la edad de 22 semanas que el feto es capaz de montar consistentemente una respuesta inmunitaria a la infección ${ }^{25}$. Los niveles de interleuquinas, interferones y del factor de necrosis tumoral son mucho más bajos en los recién nacidos prematuros que en los nacidos a término - este es un hallazgo importante dado el papel central que pueden desempeñar las citoquinas en la fisiopatología de la sífilis congénita. Sin embargo, otros componentes del sistema inmunitario - tales como la función de las células T - están lejos de ser competentes en la gestación temprana ${ }^{26}$.

Aunque aún no está claro por qué algunos fetos son más afectados que otros y hay poca información para explicar el patrón de participación, algunos trabajos de investigación nos ayudan a comprender mejor la secuencia de afectación. Hollier y colaboradores ${ }^{27}$ en estudios realizados en fetos de gestantes con sífilis encontraron que la función hepática anormal representa una primera manifestación de una verdadera infección, antes del crecimiento hepático.

El diagnóstico del daño en el Sistema Nervioso Central ha sido difícil; la interpretación de los resultados de las pruebas convencionales (VDRL en líquido cefalorraquídeo (LCR), relación proteína/ glóbulos blancos) es un problema en el recién nacido. Michelow y colaboradores. ${ }^{28}$ evaluaron 148 niños nacidos de madres con sífilis, el $64 \%$ de los cuales no habían sido tratados antes del parto. Entre los lactantes que no habían sido expuestos a antibióticos, el $22 \%$ tuvo un resultado positivo de la prueba de infectividad en conejos en LCR. Esta evidencia sugiere que el involucramiento del LCR es más probable si la madre se clasificaba como sífilis secundaria o latente temprana $(29 \%)$ que con otras clasificaciones (11\%). Además, entre los lactantes que no fueron expuestos a los antibióticos convencionales y que tenía pruebas positivas de sífilis congénita, el $41 \%$ se le había detectado treponemas en LCR.

\section{La clínica en la sífilis congénita}

La sífilis congénita puede traducirse en muerte fetal con aborto espontáneo, generalmente después del primer trimestre de gestación ${ }^{29}$, o natimuertos en un 30 a $40 \%$; o puede culminar en el parto de un recién nacido prematuro con evidentes signos clínicos de infección; o nacer completamente asintomático ${ }^{30}$. La presentación de la sífilis congénita puede ser clínicamente similar a la observada tras infección por citomegalovirus, toxoplasma o rubeola.

Cuando se produce la infección del feto, todos los órganos son afectados debido a la siembra por la diseminación de espiroquetas. Como se manifestó anteriormente, la hepatitis aparece como la más temprana manifestación y puede ser diagnosticada mediante la elevación de las transaminasas en sangre fetal; la anemia y trombocitopenia ocurre más tardíamente y se va a manifestar mediante un cuadro de hidrops fetal o edema difuso por insuficiencia cardiaca; una prueba negativa de Coombs contribuye al diagnóstico ${ }^{31}$ Puede desarrollarse también, retardo de crecimiento intrauterino ${ }^{32}$.

\section{Sífilis congénita temprana}

La hepatomegalia es el signo más frecuente en sífilis temprana. La función hepática puede ser normal pero la ictericia causada por la hepatitis sifilítica puede cursar con elevación de transaminasas y fosfatasa alcalina. Puede incrementarse la bilirrubina directa por colestasis y alargarse el tiempo de protrombina .

\begin{tabular}{|c|c|}
\hline Órgano & Características \\
\hline Placenta & Adelgazamiento de la placenta; vellositis con infiltración endovascular y perivascular \\
\hline Hígado & Inflamación en el estroma intersticial y en la red perivascular \\
\hline Pulmones & $\begin{array}{l}\text { La lesión clásica es la "neumonía alba"; el órgano es blanco amarillento, blando, firme, y alargado. El tejido } \\
\text { conectivo está incrementado }\end{array}$ \\
\hline Tracto gastrointestinal & Inflamación mucosa y submucosa e infiltración mononuclear \\
\hline Páncreas & Infiltrado inflamatorio perivascular \\
\hline Riñón & $\begin{array}{l}\text { Daño secundario por depósito de inmunocomplejos (similar a la glomerulonefritis del adulto), infiltrado infla- } \\
\text { matorio perivascular que involucra el tejido intersticial }\end{array}$ \\
\hline Sistema Nervioso Central & $\begin{array}{l}\text { Involucramiento meningeo con adelgazamiento de la meninges basilar y endoarteritis; injuria neuronal de- } \\
\text { pendiente del intenso involucramiento de los vasos sanguíneos }\end{array}$ \\
\hline Sistema óseo & $\begin{array}{l}\text { Se puede presentar osteocondritis, periostitis, y osteomielitis. Estos hallazgos pueden reflejar cambios trófi- } \\
\text { cos no específicos resultantes de la infección generalizada severa más que el efecto directo del treponema. }\end{array}$ \\
\hline
\end{tabular}


Las manifestaciones mucocutáneas se observan en el $70 \%$ al nacimiento o desarrollarse dentro de las primeras semanas de vida. Todas las lesiones mucocutáneas tienen alta carga de espiroquetas y son altamente infectantes. Los síntomas cutáneos incluyen ${ }^{33}$ :

- Pénfigo palmo-plantar: Se aprecian vesículas ampollosas en palmas y plantas, de contenido turbio y verdoso, que se rompen rápidamente dejando la dermis descubierta. La descamación se presenta entre la primera y tercera semana.

- Sifílides maculo-papulosas: Aparecen en extremidades y zonas periorificiales. Su evolución es lenta y dejan pigmentación (manchas color café con leche). Son parecidas a las lesiones de secundarismo sifilítico en el adulto.

- Condilomas: pueden surgir en las membranas mucosas o la piel de otras zonas afectadas por la humedad o la fricción

- Rágades o cicatrices peribucales y en las márgenes del ano

- Petequias cuando existe severa trombocitopenia

- Alopecia anterior.

- Paroniquia-perionixis sifilítica (generalmente respeta el pulgar).

Los síntomas mucosos incluyen:

- Rinitis o coriza sifilítica: Se observa una secreción mucohemorrágica y ulceraciones en labio superior y vestíbulo nasal. Aparece en la primera semana de vida. Tras tratamiento, éstas lesiones pueden dejar como secuelas deformidades nasales permanentes.

El involucramiento óseo ocurre en un 60 a $80 \%$ de los casos de sífilis congénita no tratada; usualmente es múltiple y simétrica, y se resuelve espontáneamente durante los primeros seis meses de vida ${ }^{34,35}$ :

- Periostitis y desmineralización cortical en las áreas de metáfisis y diáfisis de los huesos largos.

- Osteocondritis que se presenta en articulaciones principalmente rodillas, tobillos, muñecas, y codos.

- Pseudoparálisis de Parrot, debido al dolor que presenta el recién nacido por la afección ósea, lo que clínicamente se traduce en no mover la extremidad involucrada .

- Signo de Wimberger cuando la desmineralización o destrucción de la parte superior de la tibia medial metáfisis es evidente radiográficamente.

El sistema nervioso es afectado con la llamada neurosífilis la cuál es frecuentemente asintomática, y puede deberse a la diseminación que se produce después del periodo neonatal no tratado. Los hallazgos en líquido cefalo raquideo (LCR) para ser considerado neurosífilis, son una cuenta mayor de 25 células blancas por $\mathrm{mm}^{3}$ (mayor de 200 celulas mononucleares por $\mathrm{mm}^{3}$ ), y proteínas mayor de $150 \mathrm{mg} / \mathrm{dl}$ (170 mg/dl en prematuros), y la presencia de VDRL reactivo. Aunque algunas veces los resultados en LCR pueden ser negativos a pesar de la afectación neurológica. Mayormente los resultados de LCR son positivos niños sintomáticos y en un $8 \%$ de aquellos que son asintomáticos. Existen dos formas de presentación de la neurosífilis:

- Leptomeningitis sifilítica aguda, por lo general se hace evidente durante los primeros meses de vida, con signos sugestivos de meningitis bacteriana aguda ${ }^{34}$. Los resultados de LCR sugieren un proceso aséptico. Generalmente responde bien al tratamiento antibiótico ${ }^{35}$.

- Neurosífilis meningovascular crónica, generalmente se presenta al final del primer año de vida, con signos de hidrocefalia progresiva, parálisis del nervio craneal, y regresión del neurodesarrollo; el niño presenta convulsiones y se puede presentar infarto cerebral a los dos años por endarteritis sifilítica.

Otras patologías sistémicas que se presentan son:

- Esplenomegalia y periesplenitis

- Corioretinitis con tendencia al glaucoma congénito y uveítis

- Glomerulopatía luética. Generalmente de aparición tardía, de base inmunológica (depósito de complejos antígeno-anticuerpo sobre la superficie epitelial de la membrana basal del glomérulo). Las manifestaciones clínicas son las de un síndrome nefrótico o una nefritis

- Anemia, trombocitopenia, leucopenia, y leucocitosis, se presenta con relativa frecuencia. Anemia hemolítica puede persistir luego de varias semanas de un tratamiento efectivo

- Aortitis sifilítica (generalmente asintomática).

- Neumonía alba, llamada así por la coloración blanca que adquieren los pulmones por una neumonitis fibrotizante, que aparece como un infiltrado pulmonar difuso en las radiografías de lenta resolución.

- Otros hallazgos incluyen fallas en el desarrollo, pancreatitis, miocarditis, ileitis, fibrosis gastrointestinal, masas intestinales y goma pituitaria

La triada clásica de la sífilis congénita precoz incluye el pénfigo palmo-plantar, la coriza sifilítica y la hepatoesplenomegalia (Figura 1).

\section{Sífilis congénita tardía}

El tratamiento de la sífilis congénita ha disminuido mucho en el mundo la aparición de los signos de sífilis congénita tardía; cuando no se diagnóstica o trata la infección en el periodo neonatal un $40 \%$ de los niños no tratados presentarán manifestaciones de la destrucción provocada por las lesiones primarias y por lo tanto no van a ser reversibles con el tratamiento antibiótico.

Las características son:

- Nariz en silla de montar, debido a la destrucción del cartílago nasal. 

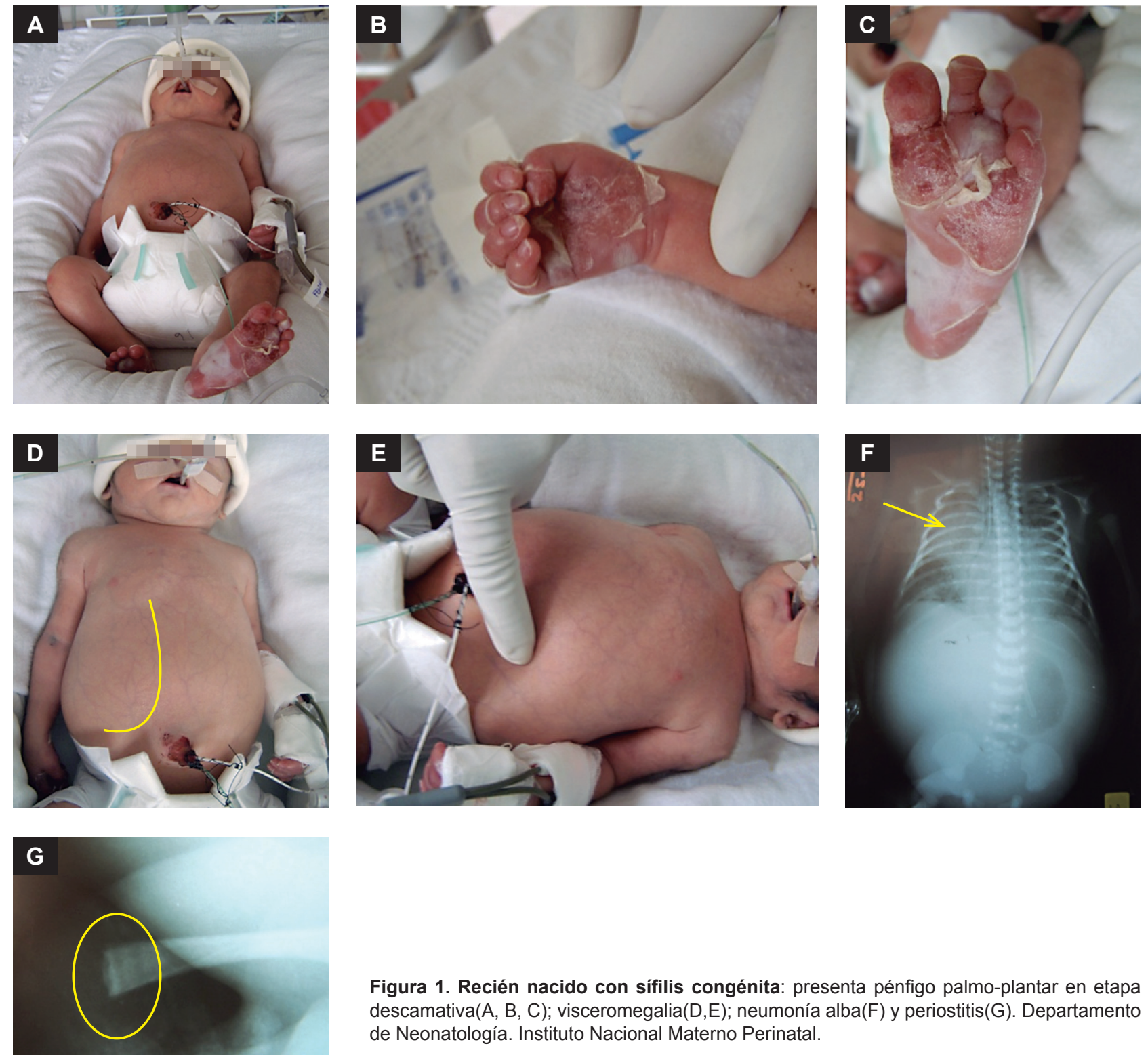

Figura 1. Recién nacido con sífilis congénita: presenta pénfigo palmo-plantar en etapa descamativa(A, B, C); visceromegalia(D,E); neumonía alba(F) y periostitis $(G)$. Departamento de Neonatología. Instituto Nacional Materno Perinatal.

- Frente olímpica, debido a la prolongada periostitis en los huesos frontales.

- Signo de Higouménakis debido al adelgazamiento de la zona esterno-clavicular del esternón

- Tibia en sable, debido al encorvamiento de la curvatura anterior de la mitad de la tibia por la periostitis.

- Articulaciones de Clutton las que son simétricas, indoloras, con derrames sinoviales estériles, normalmente localizado en las rodillas.

- Perforación de paladar duro.

- Dientes de Hutchinson, debido a la vasculitis sifilítica presente desde el nacimiento que daña el desarrollo adecuado de los dientes (en forma de clavija, incisivos con excavación central), se presenta también los molares en mora (primeros molares multicúspides).

- Sordera, por afección del octavo nervio se desarrolla en aproximadamente el 3 por ciento de los casos no tratados y, a menudo comienza con la pérdida de la audición de alta frecuencia cuando el niño tiene entre 8 y 10 años de edad.
- Queratitis intersticial (normalmente entre 5 y 20 años de edad), glaucoma secundario o cicatrices corneales.

- Atrofia óptica debido al daño retinal temprano o a la hidrocefalia.

- Rágades, grietas o fisuras en la piel que ha perdido su elasticidad, especialmente frecuentes alrededor de la boca, ano o genitales, derivados de los daños mucocutáneos al nacimiento

Los dientes de Hutchinson, la queratitis intersticial y la sordera conforman la clásica triada de Hutchinson, que caracteriza a la sífilis congénita tardía.

\section{EL TRATAMIENTO}

El diagnostico de sífilis congénita es complicado debido a la transferencia transplacentaria de anticuerpos treponémicos y no treponémicos al feto. La decision terapéutica debe realizarse basado en varios criterios 
que incluyen la identificación de la sífilis en la madre, el adecuado tratamiento materno, la presencia clínica, laboratorio o radiográfica de evidencia de sífilis en el neonato; y la comparación de los títulos serológicos no treponémicos entre la madre (en el momento del parto) y el recién nacido ${ }^{36}$. La penicilina es la única droga que se utiliza para el tratamiento del neonato con diagnóstico de sífilis, en dosis de penicilina $\mathrm{G}$ acuosa $100.000 \mathrm{u} / \mathrm{Kg} / \mathrm{d}$ endovenosa por 10 días si la enfermedad neonatal es probable o altamente probable, en un niño con madre con sífilis sin tratamiento o tratamiento inadecuado, si existe sintomatología o estigmas de sífilis congénita en el niño y si la prueba no treponémica del hijo es cuatro veces mayor que el de la madre. La penicilina $G$ benzatínica en dosis de 50,000 u/Kg intramuscular en una sola dosis, se utiliza si se tiene a un recién nacido de madre con sífilis no tratada o inadecuadamente tratada asintomático con títulos de prueba no treponémica menor de cuatro veces el título de la madre, con exámenes de laboratorio negativos, o de madre con terapia adecuada antes del embarazo y títulos de prueba no treponémica baja o estable $^{36}$.

\section{CONCLUSIÓN}

La presencia de casos de sífilis congénita continúa siendo un indicador negativo que desnuda fallas en nuestro sistema de salud. El conocimiento de su patogenia y tratamiento en el neonato solo nos permite aseverar que la mejor acción médica es prevenirla durante la vida uterina. El tamizaje en gestantes y el tratamiento oportuno han sido las piedras fundamentales en la prevención de sífilis congénita, de fácil acceso por su bajo costo y gran efectividad pero que hasta la actualidad no han logrado eliminar el problema. La falta de estudios publicados que analicen documentos y resultados asociados (pruebas y clínica) que traten de explicar esta falla es decepcionante, por lo que es necesario contar con grandes estudios prospectivos que involucren a la población materna e infantil, que analicen las intervenciones de salud pública y permitan conocer como estas influyen en el real impacto que tiene la sífilis congénita en la población infantil.

\section{REFERENCIAS BIBLIOGRÁFICAS}

1. Watts S. La plaga secreta: la sifilis en Europa occidental y Asia oriental 1492-1965. Cap 4 Epidemias y poder. Historia, Enfermedad, Imperialismo. Editorial Andrés Bello 1997

2. Hayden D. Pox: Genius, madness, and the mysteries of syphilis. 1st ed. New York: Perseus Books Group; 2003.

3. Centers for Disease Control and Prevention: Sexually transmitted disease surveillance 2002 supplement, Syphilis surveillance report. Atlanta GA, U.S. Department of Health and Human Services, Centers for Disease Control and Prevention, 2004

4. Rook A. Skin diseases caused by arthropods and other venomous and other noxious animals. In:Rook $A$ et al. Textbook of dermatology 4th ed., Blackwell, Oxford 1986, p. 1060
5. World Health Organization. The World Health Report 2005: Make Every Mother and Child Count. Geneva, Switzerland: WHO Press, 2005

6. Finelli L, Berman SM, Koumans EH, Levine WC. Congenital syphilis. Bull World Health Organ 1998; 78(Suppl 2):126-128

7. Schmid GP, Stoner B, Hawkes S, et al. The need and plan to eliminate congenital syphilis. Sex Transm Dis 2007; 34 Supplement:S5-S10

8. WHO. World Health Organization Global Burden of Disease Report. Geneva: WHO; 2002

9. Center for Disease Control. Congenital Syphilis. New York City 1986-88. MMWR 38: 825, 1989

10. Centers for Disease Control and Prevention: Sexually transmitted disease surveillance 2002 supplement, Syphilis surveillance report. Atlanta, GA, U.S. Department of Health and Human Services, Centers for Disease Control and Prevention, 2004

11. Genc M, Ledger WJ, Syphilis i pregnancy. Sexually Transmitted Infections 2000;76: 73-9

12. Hurtig AK, Nicoll A, Carne C, Lissauer E, Syphilis in pregnant women and the children in United Kingdom 1994-1997. BMJ 1998;317: 1617-19

13. Riedner G, Dehner KL, Gromyko A. Recent declines in reported syphilis rate in Eastern Europe and Central Asia: are the epidemic over? Sexually Transmitted Infections 2000; 76:363-5

14. Haaron Saalojee, Sithembiso Velaphi, Yasmin Goga. The prevention and management of congenital syphilis: an overview and recomendations. Bulletin of the World Health Organization 2004; 82:424-430

15. Valderrama J, Zacarías F, Mazin R. Sífilis Materna y Sífilis congénita en América Latina: un problema grave de solución sencilla. Revista Panamericana de salud Pública 16 (3) 2004

16. Beksinska ME, Mullick S, Kunene B, A case study of antenatal syphilis screening in South Africa: successes and challenges. Sexually Transmitted Diseases 2002; 29: 32-7

17. Centers for Disease Control and Prevention. Sexually transmitted diseases treatment guidelines 2006. MMWR Morb Mortal Wkly Rep 2006; 55 RR-11

18. Ingall D, Sanchez PJ. Syphilis. In: Remington JS, Klein JO, editors. Infectious diseases of the fetus and newborn infant. 5th ed. Philadelphia: Saunders; 2001. p. 643-81

19. Benirschke K. Syphilis - the placenta and the fetus. American Journal of Diseases of Children 1974;128:142-3.

20. Harter CA, Benirschke K. Fetal syphilis in the first trimester. American Journal of Obstetrics and Gynecology 1976;124:705-11

21. Radolf JD, Norgard MV, Brandt ME, Isaacs RD, Thompson PA, Beutler B. Lipoproteins of Borrelia burgdorferi and Treponema pallidum activate cachetin/tumor necrosis factor synthesis: analysis using a CAT reporter construct. Journal of Immunology 1991;147:1968-74.

22. Riley BS, Oppenheimer-Marks N, Hansen EJ, Radolf JD, Norgard MV. Virulent Treponema pallidum activates human vascular endothelial cells. Journal of Infectious Diseases 1992;165:484-93.

23. Norgard MV, Riley BS, Richardson JA, Radolf JD. Dermal inflammation elicited by synthetic analogs of Treponema pallidum and Borrelia burgdorferi lipoproteins. Infection and Immunity 1995;63:1507-15

24. Silverstein AM. Congenital syphilis and the timing of immunogenesis in the human fetus. Nature 1962;194:196

25. Daffos F, Forestier F, Grangeot-Keros L, Capella Pavlovsky M, Lebon P, Chartier M, et al. Prenatal diagnosis of congenital rubella. Lancet 1984;2:1-3 
26. Lewis DB, Wilson CB. Developmental immunology and role of host defenses in neonatal susceptibility to infection. In: Remington JS, Klein JO, editors. Infectious diseases of the fetus and newborn infant. 4th ed. Philadelphia: Saunders; 1995. p. 20-98.

27. Hollier LM, Harstad TW, Sanchez PJ, Twickler DM, Wendel GD Jr. Fetal syphilis: clinical and laboratory characteristics. Obstetrics and Gynecology 2001;97:947-53.

28. Michelow IC, Wendel GD Jr, Norgard MV, Zeray F, Leos NK, Alsaadi R, et al. Central nervous system infection in congenital syphilis. New England Journal of Medicine 2002;346:1792-8

29. Hook EW III, Peeling RW: Syphilis control-a continuing challenge. N Enl J Med 351:122-124, 2004

30. Jensen HB: Congenital syphilis. Semin Pediatr Infect Dis 10:183-194,1999
31. Bulova SI, Schwartz E, Harrer WV: Hydrops fetalis and congenitalsyphilis. Pediatrics 49:285-287, 1972

32. Gust, DA, Levine, WC, St Louis, ME, et al: Mortality associated with congenital syphilis in the United States, 19921998. Pediatrics 2002.

33. Wolf $\mathrm{B}$, Kalangu $\mathrm{K}$ : Congenital neurosyphilis revisited. Eur $\mathrm{J}$ Pediatr 152:493, 1993

34. Wilkinson RH, Heller RM: Congenital syphilis: Resurgence of an old problem. Pediatrics 47:27-30, 1971

35. Sánchez PJ, Gutman LT: Syphilis, in Feigin RD, Cherry JD, Demoler GJ, et al (eds): Textbook of Pediatric Infectious Diseases (5th ed.) Philadelphia, W.B. Saunders, Co., 2004, pp 1724-1743

36. CDC DoHaHs. Sexually Transmitted Diseases Treatment Guidelines, 2006 